\title{
Tipos de maltrato y estudio de sus efectos
}

\author{
Juan Francisco Muñoz Olano ${ }^{1}$
}

\begin{abstract}
This article is a review of approaches developed in scientific research to understand the effects of abuse in childhood and adolescent life, especially on cognitive, emotional and behavioural functioning. The article begins returning to the social relevance of this issue in our environment, and then address the methodological and research challenges have been own global and local level, this type of study. Next, the article takes the need for vision from the development and ecological point of view, to allow studying the psychological effects of abuse. Finally, the article focuses on recapitulate what are the main types of abuse that have been studied today, and why it is necessary to understand them from a development perspective, it is contextualized, and to resume the progress made by the studies neuro-scientific.
\end{abstract}

\author{
Editado por | William Alejandro \\ Jiménez-Jiménez \\ Universidad de Boyacá, Colombia.
}

Revisado por I Jaiver Humberto Urrego Alfaro, Universidad Manuela Beltrán, Colombia.

Keywords | Abuse, childhood, emotions, cognition, neuroscience.

\section{Introducción}

En Colombia, las cifras de maltrato infantil han llegado a números alarmantes. Entre Enero del 2012 y Enero del 2013 el Instituto Colombiano de Bienestar Familiar (ICBF) registró 16.457 casos de niños maltratados, a quienes fue necesario abrirles procesos legales para el restablecimiento de sus derechos, siendo necesario además que un $34 \%$ de estos infantes, entre los seis y los doce años de edad, ingresaran a instituciones de protección (Instituto Colombiano de Bienestar Familiar, 2014). La intervención que Estado y sociedad deben llevar a cabo para favorecer la condición de los infantes está consagrada en la Constitución Colombiana de 1991: "Los derechos de los niños prevalecen sobre los de los demás" (Constitución de la República de Colombia, en prensa); como un deber ser del Estado y la sociedad, la carta magna propende

\footnotetext{
${ }^{1}$ Juan Francisco Muñoz Olano Docente de tiempo completo del programa de Psicología. Universidad de Boyacá.

[囚] Email: jfmunoz@uniboyaca.edu.co
}

$\boldsymbol{\epsilon} \| 92$ 
porque se cumplan los derechos fundamentales de niños, niñas y jóvenes, referentes a la dignidad, la integridad y el bienestar.

Sin embargo, cada vez se hacen más necesarios los desarrollos de estrategias y métodos de investigación precisos y efectivos que permitan identificar los casos de maltrato infantil y las secuelas que estos eventos suelen dejar en los infantes. Esta tarea científica, de poder medir los casos y el impacto del maltrato en un gran porcentaje de la población infantil, ha sido particularmente difícil en Colombia, debido a los costos y las técnicas que requiere. Como principales dificultades se encuentran: El no contar con pruebas psicológicas que estén validadas y presenten medidas de confiabilidad en el medio local, así como el no contar con un número significativo de casos identificados para poder evaluar muestras significativas (Ramírez, 2006).

\section{Dificultades metodológicas del estudio de los efectos del maltrato infantil y adolescente}

Como dificultades metodológicas en el estudio del maltrato infantil, se han presentado varias: saber con precisión si un padre maltratado será maltratador, o si un padre maltratador fue maltratado en su infancia, siempre son hipótesis consideradas, que tampoco han sido claramente evidenciadas (Belsky, 1993). Desde años anteriores, se han planteado dificultades en el estudio de los efectos del maltrato, siendo que no se ha podido constatar que padres que sufrieron experiencias de negligencia y maltrato físico en su infancia temprana puedan recordar con claridad estos eventos. Para ver si se relacionan con su propia conducta como padres (Golwdyns, 1984; citado en Belsky, 1993); en investigaciones pasadas, también se encontraron inconsistencias en los casos que planteaban relaciones entre problemas de autoestima, depresión y ansiedad con el hecho de convertirse en maltratador, así como con el que los padres que ejercían maltrato hubiesen sido maltratados en su propia infancia (Lawson \& Hays, 1989; citados por Belsky, 1993). Importante considerar, además, que como ha planteado más recientemente Marshall (2012), ha existido una dificultad para evidenciar los síntomas del maltrato, diferenciar entre el maltrato físico, el abuso sexual y el abuso emocional. Sin embargo, se han llevado a cabo investigaciones que plantean corregir estas dificultades. Heyman y Smith (2006) desarrollaron un estudio con una serie de pasos para poder clarificar con mayor precisión cómo diferenciar los tipos de maltrato y sus efectos en los infantes y en los adultos, considerando que el principal problema estaba en no definir claramente las características de cada tipo de maltrato. Primero que nada, los investigadores llevaron a cabo un estudio de validez de contenido sobre las definiciones de 
varios tipos de maltrato, entrevistando a expertos clínicos. Segundo, los investigadores llevaron a cabo una conceptualización sobre cómo eran los diferentes tipos de maltrato y cómo era el comportamiento de los maltratadores, incluyendo un análisis de qué comportamientos se omiten, y no solo cuáles se llevan a cabo. Y como tercer paso, los investigadores desarrollaron una operacionalización de los tipos de maltrato, siguiendo también los criterios establecidos por ley en los Estados Unidos, para especificar cuándo hay maltrato. En los resultados, se encontró mayor claridad en los diagnósticos de abuso sexual, maltrato físico y negligencia, que en los de abuso emocional o psicológico. De hecho, la diferenciación entre condiciones de solo abuso emocional fue difícil de establecer con respecto a los otros tipos de maltrato.

Una dificultad metodológica inherente al estudio de los efectos del maltrato también ha estado en la dificultad para llevar a cabo estudios longitudinales a través de años, que evidencien las consecuencias progresivas del maltrato en el desarrollo psicológico, razón por la cual se han llevado a cabo más estudios de tipo retrospectivo, donde padres maltratadores y personas maltratadas han compartido testimonios sobre sus historias de vida y han relacionado dichas historias con su funcionamiento psicológico actual (Tajima, Herrenkohl, Huang y Whitney, 2004). Sin embargo, como lo plantean los mismos autores (Ibídem), la validez de estos estudios ha sido cuestionada. Los recuerdos de personas que sufrieron de maltrato suelen ser distantes y tienden a fallar (Henry, 2004; citado por Tajima et al, 2004), sobre todo siendo que diversos estudios han usado diferentes formas de medición de los recuerdos retrospectivos. Una definición clara de los eventos de maltrato se hace más difícil teniendo presente el impacto de estos fallos de la memoria.

\section{Una perspectiva desde el desarrollo y la visión ecológica}

Los estudios muestran una estrecha relación entre haber sufrido condiciones de maltrato en la infancia y la adolescencia (abuso sexual, maltrato físico, negligencia, maltrato psicológico o emocional), y el desarrollo de problemas emocionales, cognitivos y de conducta en infantes, adolescentes y adultos. Con el trascurrir de los años en deficiencias cognitivas (Nikulina \& Widom, 2013; Delima \& Vampini, 2011; Kinard, 2004); en el desarrollo de problemas emocionales y de comportamiento (Jaffe \& Kohn, 2011; Delima \& Vampini, 2011; Barrera, 2007). Sin embargo, teorías basadas en el impacto del aprendizaje social y del castigo han hecho especial énfasis en el efecto de experiencias directas sobre quienes se convierten en personas maltratadoras

\section{$\boldsymbol{E} \mid 94$}


de sus hijos (Belsky, 1993). Pero esta explicación no da razón del por qué hay padres que no son maltratadores, aunque tuvieron infancias en las que recibieron estos tratos, como así mismo hay padres que pueden ser maltratadores físicos, cuando fueron víctimas de negligencia o abuso emocional, mas no de maltrato físico directamente. La razón del por qué las experiencias directas no explican claramente la adquisición de un patrón de maltrato por parte de un padre, haría necesaria una visión que explique los orígenes del maltrato desde una perspectiva del desarrollo, que combine el análisis del posible efecto de experiencias directas, con el desarrollo de vulnerabilidades personales y disposiciones de acción, no directamente reforzadas o directamente instruidas en el pasado de una persona maltratadora. Estas diferencias entre posibles maltratadores se explicarían no solo por las historias reales de las personas, sino también por la "elaboración" psicológica que las personas logran hacer a través de su vida, sobre sus experiencias en la infancia y en la adolescencia; como plantearon hace ya varias décadas Main y Goldwyn (1984; citados por Belsky, 1993), las madres que recordaban a sus propias madres como distantes y su infancia como difícil, pero que con el tiempo habían logrado formarse una percepción sobre sí mismas como coherente con ser una madre cariñosa y próxima, no desarrollaban conductas de rechazo hacia sus hijos.

Una visión centrada en el desarrollo de vulnerabilidades, haría entonces necesario adoptar un punto de vista más amplio, más ecológico. Como planteó Belsky hace ya varios años:

"... el estudio ecológico del desarrollo humano... conceptualiza el maltrato infantil como un fenómeno psico-social determinado de forma múltiple por fuerzas que trabajan en el nivel individual y familiar, así como en el nivel comunitario y cultural, en los cuales, tanto el indivi-duo como la familia están involucrados". (Belsky, 1980, p.320).

Una visión más ecológica explica por qué una persona maltratadora lo puede ser por múltiples razones y por el efecto de múltiples variables. Entre estas múltiples variables, resaltan variables familiares, individuales y contextuales.

Entre las variables familiares, se encuentran la transmisión inter-generacional de la predisposición al maltrato (Rosenberg, 1987); esta transmisión inter-generacional se refiere a como las acciones negligentes o aversivas de los padres pueden generar patrones de respuesta emo- 
cional y conductual en los hijos que los predisponen a ser adultos maltratadores, cuando experiencias de abuso o negligencia afectan sus circuitos neurales de respuesta ante el estrés (Teicher, 2002; citado por Degregorio, 2012). Sin embargo, para que se desarrolle un patrón maltratador, parece ser necesario que estas experiencias tempranas se den junto con una vulnerabilidad genética. Jaffe (2004) encontró, al hacer estudios de comparación entre gemelos, que los gemelos monocigóticos tendían a ser disciplinados de una manera más similar que la dada entre gemelos dicigóticos. Esto en tanto los gemelos monocigóticos presentaban mayores similitudes temperamentales y de personalidad, que mediaban la conducta de crianza de los padres hacia ellos; como una variable familiar, también es importante tener presente el historial de consumo de alcohol y sustancias psicoactivas dado en una familia. Estudios sobre los efectos del alcoholismo y la drogadicción en los padres han evidenciado que estos problemas de consumo incrementan la probabilidad de maltrato (Muller, Fitzgerald, Sullivan \& Sucker, 1994; Locke \& Newcomb, 2003). Sin embargo, se ha evidenciado que el maltrato puede encontrarse tanto en casos relacionados con el alcoholismo de forma independiente a la drogradicción (Locke \& Newcomb, 2003), siendo más probable por parte de los hombres, cuando el medio ambiente representa condiciones de estrés y alienación social, mientras que para las mujeres, solo cuando el ambiente en el que viven presenta condiciones de alienación social (Muller et al, 1994). Importante también los hallazgos sobre cómo la percepción de los padres sobre sus hijos puede influenciar los estilos de crianza y hacerlo maltratadores, cuando el hijo tiene un temperamento que hace más probable que el padre vea como necesarias las prácticas maltratadoras (Gracia, 2002).

Entre las variables individuales, resaltan principalmente la edad en la cual se experimentan por primera vez las situaciones de maltrato, así como el género. Kaplow y Widom (2007) encontraron que una experiencia temprana de maltrato, dada entre los años pre-escolares y escolares, se asocia más con el desarrollo de problemas de ansiedad y depresión, mientras que una experiencia más tardía de maltrato, entre la edad escolar y la adolescencia, se asocia más con el desarrollo de problemas conductuales, como el comportamiento desajustado socialmente. McCleod, Ferguson y Horwood (2014) encontraron que personas que reportaron haber sido maltratadas físicamente presentaban, en mayor proporción, problemas de relación con sus parejas, treinta años después de los eventos de maltrato. El género también evidencia importantes aspectos a tener presentes a nivel individual. Muller (1995) encontró de una muestra de

\section{$\boldsymbol{\epsilon} \| 96$}


mil quinientos padres entrevistados, que la mayoría de ellos recibieron en mayor proporción acciones maltratadoras por parte de los padres de su mismo género.

Entre las variables contextuales, es importante tener presente aquellas que resaltaba Rosenberg décadas anteriores:

\begin{abstract}
"Aplicar la perspectiva ecológica implica atender a contextos fuera de la familia en los cuales el maltrato psicológico ocurra, donde además se debe considerar a la comunidad y las actitudes de la sociedad, y cómo éstas influencian el valor psicológico de los niños y de las prácticas de cuidado pensadas para contribuir en la salud mental". (Rosenberg, 1987, p.166).
\end{abstract}

El ambiente o contexto, también se refiere a variables culturales como la crianza religiosa y el nivel socio-económico. Es así como en un estudio realizado en Israel (Ben-Arieh, 2010) evidenció que entre más religiosas son las familias, mayor proporción de maltrato se encuentra en ellas. En el mismo estudio, también se encontró que a mayor tasa de desempleo en un barrio, mayor taza de maltrato se presentan en las familias.

Una serie de estrategias novedosas se han desarrollado para poder combinar un estudio conjunto de los factores familiares, individuales y contextuales del maltrato. Oberlander, Thompson, Lewis, Proctor, Isbel, Duvowitz, Litrownick y Black (2011) Ilevaron a cabo una investigación de seguimiento a 637 casos que reportaron historiales de abuso sexual, maltrato físico y negligencia, donde pusieron a prueba el efecto de la monitorización que hacían los padres y los jóvenes sobre su propio comportamiento, todos los días. Los padres compartían un reporte sobre su forma de criar a sus hijos, y los hijos, sobre su forma de comportarse. Este estudio evidenció una notable reducción del riesgo de abuso sexual y emocional hacia las hijas. En otro estudio, el uso de un método de análisis de datos de manera personalizada en un grupo de 788 jóvenes con diversos historiales de maltrato vividos, permitió evidenciar que aquellos que sufrieron maltrato físico desarrollaron más problemas de conducta, mientras que aquellos que sufrieron formas combinadas de maltrato por abuso sexual y negligencia, sufrieron condiciones de victimización que sólo los hicieron más vulnerables a más eventos de maltrato, transcurrido el tiempo (Villodas, Litrownik, Thomson, Roesch, English, Dubowitz, Kotch y Runyan, 2012). Las innovaciones dadas con estos estudios evidencian importantes desarrollos por venir. 
Es así como, la complejidad dada en medir las historias de maltrato que han sufrido infantes y adultos a través de su historia de vida, se ha convertido en una de las principales dificultades para el estudio del impacto que el maltrato puede tener en el funcionamiento psicológico, principalmente, en los niveles cognitivo, emocional y conductual. Se hace entonces necesaria la clarificación de los diferentes tipos de maltratos existentes, así como, la explicación de los mecanismos de maltrato que afectan el funcionamiento cognitivo, emocional y personal.

\section{El estudio de los efectos del maltrato infantil}

En investigaciones de años anteriores, autores como Rosenberg (1987) resaltó la dificultad en poder medir y evaluar la presencia de casos de maltrato psicológico, así como poder hacerle un seguimiento a casos de maltrato sexual y físico. Sin embargo, en años más recientes ha avanzado el estudio de los efectos del maltrato en el funcionamiento cerebral y neurofisiológico, así mismo como el estudio del desempeño de infantes con historiales de maltrato en tareas comportamentales y cognitivas. Es el caso de los hallazgos encontrados por Nikulina y Widom (2013), quienes evidenciaron que infantes con historiales de abuso sexual y víctimas por negligencia de los padres, mantenían al largo plazo deficiencias en funciones cognitivas de tipo ejecutivo, involucradas en la realización de tareas de razonamiento no verbal. Así mismo, Moreno (en prensa) evidenció un decremento en las habilidades verbales de infantes víctimas de abandono y abuso emocional. Los investigadores Jaffe y Kohn (2011) encontraron que niños con antecedentes de maltrato, sea físico, por negligencia o por abuso sexual, desarrollaban en mayor proporción problemas tanto de conducta, como emocionales, que infantes sin estos historiales. Estos problemas incluían, según los autores, problemas de ansiedad, de depresión y de angustia, así como problemas de control de impulsos, agresividad y comportamientos poco pro-sociales. El estudio de Barrera (2009), realizado en Colombia, evidenció que el 30\% de una muestra de 38 infantes con historial de abuso sexual presentaban síntomas de estrés pos trauma. A nivel neurofisiológico, Delima y Vampini (2011) encontraron que niños con historial de maltrato presentan particularidades en el funcionamiento de sus circuitos neurales directamente regulación del comportamiento y de respuesta emocional ante el estrés.

A continuación se revisan conceptos, hallazgos y teorías que explican algunas de las particularidades que hoy en día se conocen sobre el efecto del maltrato infantil en el funcionamiento psicológico. Importante, empezar por clarificar los diferentes tipos de maltrato existentes. 


\section{Tipos de maltrato infantil}

El maltrato infantil tiene efectos en la dinámica cerebral, que dejan huella en el funcionamiento cognitivo y emocional. Estos efectos, se ha comprobado (Delima y Vampini, 2011), tienen que ver con cambios funcionales y anatómicos de la respuesta del cerebro ante el estrés emocional causado por dichos eventos. Sin embargo, los efectos no son siempre iguales, sino que dependen de la edad del infante, en la cual es víctima de maltrato, así como de la persistencia y el tipo de perpetrador de la misma. Es por esto que, a continuación, se ilustra el efecto de estas variables, empezando por identificar los diferentes tipos de maltrato existentes, sus efectos diferenciales en la cognición y las emociones, para posteriormente, abordar los cambios neurofisiológicos en el cerebro, que explicarían los cambios cognitivos y emocionales, específicamente, en funciones como la memoria, la atención y las funciones ejecutivas. Además de los cambios específicos en la respuesta emocional consciente e inconsciente, dados por diferentes mecanismos de aprendizaje ante el trauma y el condicionamiento emocional.

Kinard (2004) plantea los siguientes tipos de maltrato:

1. Maltrato físico: Caracterizado por las presencias de heridas (mayores o menores), moretones, marcas, laceraciones, fracturas, heridas internas, quemaduras y heridas en la cabeza. Sus efectos se asocian más con comportamiento poco cooperativo por parte de los infantes hacia otros, así como con menos empatía y menor compromiso emocional con las otras personas. Además, el maltrato físico se ha asociado con problemas en la inhibición de respuestas (problemas externalizantes), que suelen conducir a expresiones de rabia y frustración sin control. El maltrato físico crónico se ha asociado con el desarrollo de personalidad antisocial (Kinard, 2004).

2. Negligencia: Consiste en situaciones como el no proveer de comida apropiada al infante, ni brindarle refugio, ni ropa. Mantener al infante en condiciones precarias de higiene y salud. Que el cuidador no esté pendiente de supervisar al infante y educarlo. Sus efectos están asociados con síntomas depresivos y conductas suicidas (problemas internalizantes), así como bajos índices de coeficiente intelectual. La negligencia prolongada durante varias etapas del desarrollo se ha asociado con problemas de conducta anti-social (Kinard, 2004). 
3. Abuso sexual: Consiste en la explotación por parte del cuidador o adulto sobre el niño, con abuso o acoso, que involucra relaciones sexuales, maltrato sexual y abuso emocional. Sus efectos están asociados con el estrés pos-trauma y dificultades atencionales. EI ser víctima de abuso sexual se ha asociado con la posibilidad de desarrollar un trastorno limítrofe de la personalidad (Kinard, 2004).

4. Maltrato o abuso emocional o psicológico: De acuerdo con Garbarino (1986; citado por Gracia, 1993), el maltrato psicológico o emocional consiste en un patrón de conductas psicológicamente destructivas, caracterizado por actos $u$ omisiones de un adulto que amenaza el desarrollo de la identidad y la competencia social de un menor. Puede tomar las siguientes cinco formas: maltrato por rechazo, donde el adulto rechaza el reconocer la importancia y la legitimidad de las necesidades de un menor; maltrato por aislamiento, donde el adulto impide al menor el acceso a experiencias sociales habituales y normales, impidiendo la formación de amistades, haciéndole creer que está solo en el mundo; maltrato aterrorizando, donde el adulto ataca verbalmente al menor, crea un clima de miedo, intimida, asusta al menor, y le hace creer que el mundo es un lugar caprichoso y hostil; maltrato por ignorar, donde el adulto no proporciona la estimulación y responsabilidad esencial para su desarrollo, impidiendo su crecimiento emocional y su desarrollo intelectual; maltrato por corrupción, donde el adulto desocializa al menor, le estimula a implicarse en conductas contrarias a una moral socializada y estándar. Los efectos del maltrato psicológico todavía no son concluyentes (Kinard, 2004).

Los diferentes tipos de maltrato varían de acuerdo a su severidad, su cronicidad, su patrón de ocurrencia y los perpetradores del mismo (Kinard, 2004). Para poder estimar el impacto de estas variables, es necesario tener presentes los diferentes estadios del desarrollo infantil, siendo que dependiendo de en qué momento del desarrollo ocurra el maltrato y qué tanto se repite en diferentes estados, entonces, las secuelas serán distintas.

\section{El maltrato infantil analizado desde las etapas del desarrollo infantil}

Según Erikson (1950; última edición, 2000), los estados del desarrollo son: Infancia (0-18 meses), niñez temprana (18 meses - 3 años), etapa pre-escolar (de los 3 a los 6 años), y los pri- 
meros años escolares (de los 6 a los 9 años). Estos estados del desarrollo serán retomados a continuación, para analizar el impacto de los diferentes tipos de maltrato.

El abuso sexual, perpetuado más frecuentemente por alguien fuera de la familia, tiene un gran impacto cuando ocurre en cualquier estado del desarrollo. Aunque este tipo de maltrato suele ocurrir algunas veces, o incluso solo una, es el que más impacto emocional tiene a lo largo de la vida. Por el lado del maltrato físico, suele ser perseverante, y ocurrir más frecuentemente entre la etapa pre-escolar y la de los primeros años escolares (2 etapas). Sin embargo, su continuidad en el estado de los primeros años escolares suele generar mayores efectos cognitivos y emocionales. Este maltrato suele ser perpetrado más por familiares que por extraños; en el caso de la negligencia, al igual que el maltrato físico, suele tener como responsables más a los familiares y conocidos, y suele generar más efectos cognitivos y emocionales cuando se perpetúa desde la niñez temprana hasta los primeros años escolares (3 etapas). Es interesante tener presente que el mayor impacto del maltrato físico y la negligencia, a nivel consciente, viene dado porque se mantienen entre dos y tres etapas del desarrollo temprano. De hecho, es su continuidad entre estas etapas del desarrollo lo que genera peor pronóstico. Si estos tipos de maltrato sólo se dan entre las etapas de infancia y niñez temprana, pero no continúan en la etapa de pre-escolar, los infantes pueden desarrollar condiciones de resiliencia (Chorpita y Barlow, 1998) que los hacen recuperarse de estas situaciones. Sin embargo, para el caso del abuso sexual, no importa la etapa del desarrollo y si éste continúa o no, aun así suele tener graves repercusiones emocionales. Esta diferencia podría explicarse por un impacto distinto entre los tipos de maltrato, siendo que el maltrato físico y la negligencia dejan secuelas conscientes, mientas que el abuso sexual suele dejar secuelas inconscientes, y esto debido a la coexistencia de mecanismos de aprendizaje emocional tanto conscientes como inconscientes en el cerebro (Gluck, 2009). 


\begin{tabular}{|c|c|c|c|}
\hline $\begin{array}{l}\text { Tipo de } \\
\text { maltrato }\end{array}$ & Ocurrencia y cronicidad & Perpetrador & $\begin{array}{c}\text { Efectos a lo largo del desarrollo } \\
\text { personal }\end{array}$ \\
\hline $\begin{array}{l}\text { Abuso } \\
\text { sexual }\end{array}$ & $\begin{array}{l}\text { Los efectos crónicos se } \\
\text { desarrollan con solo una o dos } \\
\text { veces que ocurra. }\end{array}$ & $\begin{array}{l}\text { Más probable que sea } \\
\text { un familiar o persona } \\
\text { cercana. }\end{array}$ & $\begin{array}{l}\text { Probabilidad de desarrollar estrés pos } \\
\text { trauma }{ }^{1} \text { como respuesta condicionada } \\
\text { emocionalmente a una o solo dos } \\
\text { ocurrencias del evento; desarrollo de } \\
\text { féficits de atención; probabilidad de } \\
\text { desarrollar una personalidad limítrofe }{ }^{2} \text {. }\end{array}$ \\
\hline $\begin{array}{l}\text { Maltrato } \\
\text { físico }\end{array}$ & $\begin{array}{l}\text { Efectos crónicos ocurren cuando } \\
\text { el maltrato físico se repite } \\
\text { durante dos o más etapas del } \\
\text { desarrollo: De la etapa pre- } \\
\text { escolar a los primeros años } \\
\text { escolares (Ej. de los } 2 \text { a los } 5 \text { años, } \\
\text { aproximadamente); durante los } \\
\text { años escolares (Ej. de los } 5 \text { a los } \\
10 \text { años, aproximadamente). }\end{array}$ & $\begin{array}{l}\text { Es más probable que } \\
\text { el perpetrador sea un } \\
\text { miembro de la familia. }\end{array}$ & $\begin{array}{l}\text { Disminución del comportamiento } \\
\text { cooperativo, de las respuestas de } \\
\text { empatía y del compromiso emocional } \\
\text { ante otras personas. Dificultades en la } \\
\text { inhibición de respuestas impulsivas. Su } \\
\text { efecto se ha asociado con la posibilidad } \\
\text { de desarrollar una personalidad de tipo } \\
\text { anti social }{ }^{3} \text {. }\end{array}$ \\
\hline Negligencia & $\begin{array}{l}\text { Los efectos crónicos suceden en } \\
\text { condiciones similares a las que } \\
\text { ocurren cuando el maltrato físico } \\
\text { tiene efectos crónicos. }\end{array}$ & $\begin{array}{l}\text { Suele ser causado por } \\
\text { un cuidador, como el } \\
\text { padre y/o la madre, los } \\
\text { hermanos y familiares } \\
\text { cercanos. }\end{array}$ & $\begin{array}{l}\text { Sus efectos, se ha evidenciado, se asocian } \\
\text { con el desarrollo de síntomas depresivos } \\
\text { y de aumento de la posibilidad de } \\
\text { conductas suicidas. Sus efectos crónicos } \\
\text { también se asocian con el desarrollo de } \\
\text { una personalidad anti social y con déficits } \\
\text { en el nivel intelectual (IQ). }\end{array}$ \\
\hline $\begin{array}{l}\text { Maltrato } \\
\text { psicológico }\end{array}$ & $\begin{array}{l}\text { Efectos crónicos durante varias } \\
\text { etapas del desarrollo. }\end{array}$ & $\begin{array}{l}\text { Suele ser causado por } \\
\text { padres, hermanos, } \\
\text { familiares cercanos, } \\
\text { figuras de autoridad y } \\
\text { compañeros. }\end{array}$ & $\begin{array}{l}\text { No se han determinado con claridad los } \\
\text { efectos que genera, pero se ha asociado } \\
\text { con el desarrollo de problemas de } \\
\text { autoestima. }\end{array}$ \\
\hline
\end{tabular}

Tabla 1 / Variables implicadas en la cronicidad y los efectos de los cuatro tipos de maltrato infantil. 
Importante además tener presente el impacto del maltrato a lo largo de varias etapas del desarrollo (Ver Tabla 1). Según investigaciones de Jaffe y Kohn (2011), los niños víctimas de maltrato continuo y extensivo tienen más problemas internalizantes y externalizantes, además de presentar menores puntuaciones en pruebas de coeficiente intelectual (IQ). Sin embargo, la permanencia del maltrato en varias etapas del desarrollo no ha podido asociarse con cambios en el comportamiento pro-social. Además, los autores también encontraron que si el maltrato ocurre por primera vez entre los 6 y 9 años de edad, y se mantienen de forma crónica y continua, el infante puede terminar muy afectado por esta condición. Como puede verse en la tabla 1 , la cronicidad debe analizarse dependiendo de las etapas del desarrollo involucradas.

\section{Efectos del maltrato}

Es así como Jaffe y Kohn (2011) realizaron un estudio que identificó los efectos de los diferentes tipos de maltrato en los procesos cognitivos y emocionales de infantes y adolescentes. Como principal resultado, encontraron que los niños maltratados en múltiples periodos de su desarrollo tienen más problemas emocionales y de conducta de tipo internalizantes (ansiedad, depresión, angustia) y externalizantes (bajo control de impulsos, agresión, menos conducta pro-social), y a su vez, presentan puntajes de coeficiente intelectual (IQ) menores a los de niños de sus mismas edades quienes no recibieron maltrato. En los resultados se encontró que cuando el maltrato había sido crónico y continuo, los infantes presentaban menores puntuaciones en IQ y más problemas internalizantes, y no solamente problemas externalizantes. Esta investigación también encontró que si el maltrato ocurrió por primera vez entre los 6 y 9 años, entonces los efectos se hacen mayores.

Como se mencionaba anteriormente, Barrera (2007) encontró presencia de estrés pos-trauma en cerca del $30 \%$ de una muestra de 38 niños con antecedentes de abuso sexual, además de una sintomatología psicopatológica variada (depresión y problemas para comunicarse). En la evaluación neuropsicológica Barrera también encontró diferencias significativas en memoria a corto plazo, dificultades en la comprensión de instrucciones y alteraciones para inhibir respuestas automáticas. La evaluación mostró en general un rendimiento inferior del grupo con antecedentes de abuso sexual.

Importante resaltar que Delima y Vampini (2011) comprobaron cambios neuro-anatómicos y fisiológicos en los cerebros de infantes que habían sido maltratados, no evidenciados en 
condiciones de desarrollo donde no se ha presentado maltrato. Como principales resultados, encontraron activaciones prolongadas del eje hipotalámico-pituitario-adrenal (HPA), lo cual parece, según los autores, afecta los circuitos neurales que comunican a la corteza pre-frontal, el hipocampo y el cuerpo calloso, generando problemas de conducta externalizantes (por una pobre auto-regulación de la conducta), junto con problemas internalizantes, como lo son los altos niveles de ansiedad. Las fallas en estas conexiones, se encontró, son más frecuentes cuando el maltrato ha sido continuo después de los 6 y 7 años, y alcanza a afectar la proliferación de neuronas y axones que conectan a estas estructuras cerebrales, implicando el desarrollo de procesos de inhibición conductual, de los cuales son responsables dichas estructuras. Esta combinación de problemas externalizantes con internalizantes, podría explicar el origen de problemas relacionados con la agresión, el control de impulsos y las tendencias suicidas que suelen presentarse en las personas que fueron víctimas de maltrato continuo durante varias fases de su desarrollo. Los efectos de los cambios neurofisiológicos también fueron evidenciados por Beach y Brody (2010) al comprobar que cambios en la actividad neurotransmisora (la recepción de la monoamino-oxidasa en la corteza cerebral), se daban en infantes que habían experimentado maltrato físico y por negligencia, el cual se asoció a su vez con que estos infantes presentaran en mayor proporción conductas antisociales.

Condiciones como el abuso sexual, la negligencia y el maltrato físico pueden dejar huellas en el funcionamiento neurofisiológico de un infante, sobre todo, cuando estos eventos ocurren en etapas críticas del desarrollo infantil y se hacen continuos y extensivos en el tiempo. La mente humana siempre está tratando de predecir lo que va a ocurrir. Estos mecanismos de predicción ponen en funcionamiento formas de aprendizaje de las experiencias, que tienen un impacto en las emociones, los recuerdos y las habilidades de los sujetos. La respuesta ante lo estresante, peligroso o dañino es en sí misma una habilidad, que la mayoría de las veces es automática e inconsciente, pero en muchas ocasiones también requiere de una elaboración consciente, deliberada y esforzada. Cuando es automática e inconsciente, involucra exclusivamente estructuras cerebrales, como las del cerebro medio (que incluye al tallo cerebral, el hipotálamo y la hipófisis), estructuras claves en el desarrollo de conductas necesarias para la supervivencia, como son las de dolor, huida y lucha, además de estructuras del sistema límbico, como los cuerpos mamilares, el fornix y las cortezas entorrinales y perirrinales, que se han visto involucradas en el procesamiento afectivo inconsciente de las experiencias (Gluck, 2009). 
Así mismo, cuando la respuesta ante lo estresante requiere de tomar decisiones conscientes, elaborar recuerdos episódicos y planear acciones futuras, para que dicho evento no vuelva a ocurrir, se ven involucradas estructuras cerebrales como la amígdala y la corteza del cíngulo (del sistema límbico), el hipocampo (que procesa información consciente y episódica), el cortex cerebral (principalmente el lóbulo frontal y pre-fronal, donde los mecanismos atencionales y de función ejecutiva captan lo sucedido), y el cuerpo calloso, que permite una integración entre la actividad de ambos hemisferios cerebrales, para poder responder conscientemente y de forma habilidosa ante la situación estresante.

Estos dos mecanismos (automático y consciente) suelen verse afectados ante experiencias de maltrato, debido al efecto que tiene la respuesta automática ante el estrés, en su producción de hormonas y la respuesta simpática que genera, sobre las estructuras que permiten la respuesta deliberada y consciente. Es así como la exposición traumática ante un evento (por ejemplo en el abuso sexual), o la exposición continua ante eventos aversivos (por ejemplo en el maltrato físico y ante la negligencia de los cuidadores), genera una activación prolongada del eje HPA (desequilibrando la producción de hormonas como el cortisol). Alink, Kim, Cicchetti y Rogosh (2012) encontraron que el maltrato en los niños incrementa las dificultades de interacción social asociadas a una desregulación de la hormona cortisol en el organismo de los infantes. De una muestra de 236 infantes con historiales de maltrato, se encontró que aquellos que tenían dificultades sociales por presentar comportamientos poco cooperativos asociados a sus historias de maltrato, presentaban niveles bajos de cortisol hasta después de un año de iniciar la medición, mientras que aquellos infantes que presentaban dificultades dadas por desarrollar altos niveles de ansiedad y depresión, como resultado de sus experiencias, presentaban altos niveles de cortisol, también hasta un año después de iniciar la medición de los niveles de cortisol en sus organismos.

Síntomas característicos del maltrato, como lo son los trastornos de ansiedad, las depresiones, los comportamientos suicidas, los comportamientos anti-sociales, el bajo nivel intelectual, los problemas de aprendizaje, las disfunciones sexuales, la baja autoestima, entre otros, podrían ser el resultado de estos efectos neurofisiológicos en el cerebro infantil. Sin embargo, estos efectos se hacen más claros a través de los años de maduración, algunos incluso hasta la vida adulta. Es por esto que identificar cambios en procesos cognitivos y emocionales básicos podría 
apoyar una identificación temprana de estos efectos, para orientar acciones de intervención adecuadas para estos infantes.

\section{Discusión}

La dificultad comentada inicialmente, sobre cómo medir y evaluar los efectos del maltrato infantil, requiere especificar qué tipos de maltrato infantil existen, cómo se definen y qué impacto crónico pueden generar. En la revisión hecha, se encontró que estudios empíricos soportan la medición del impacto de los tipos conocidos como maltrato físico, abuso sexual y negligencia, estando en discusión la descripción y la medición del efecto del maltrato o abuso psicológico o emocional, descrito por algunos autores. Esto en tanto que el maltrato psicológico no cuenta con causas claramente descritas y medidas, sobre cuándo empieza, cómo ocurre y cómo se evidencia en el comportamiento del perpetrador y en sus efectos en el infante. Garbarino (1986; citado por Gracia, 1993) resalta que el maltrato psicológico se puede evidenciar por conductas del perpetrador, que van del rechazo y el terror, a ignorar al infante. Pero, tales categorías resultan difíciles de definir y medir.

Sin embargo, como muestra el estudio de Kinard (2004), se han diferenciado los efectos que el abuso sexual, el maltrato físico y la negligencia pueden llegar a tener, a través de etapas del desarrollo infantil. Es así como el efecto del maltrato físico se explica por las condiciones de variables como el número de etapas en que se experimenta, la continuidad y la discontinuidad o continuidad de las mismas. Por ejemplo, un infante que sufre de maltrato físico durante la etapa pre-escolar (de los 3 a los 6 años), pero luego deja de recibir este maltrato, sufrirá menos secuelas emocionales, cognitivas y desarrollará menos problemas de comportamiento que un infante que sufre de este maltrato tanto durante la etapa pre-escolar (de los 3 a los 6 años), como durante la etapa escolar (de los 6 a los 9 años). Pero además, los efectos del maltrato físico pueden sumarse a través de etapas discontinuas. Por ejemplo, un infante que recibió maltrato físico en su primera infancia (de los 0 a los 18 meses), y luego volvió a recibirlo hasta la etapa escolar, podría estar en mayor riesgo que un infante que solo lo recibió durante la etapa escolar. Es así como el grado de efecto del maltrato físico dependería del historial de vivencia del mismo. Para el caso de los efectos de la negligencia, sucede algo similar. La negligencia suele tener secuelas con efectos crónicos en el comportamiento, la emoción y el desarrollo cognitivo, es cuando se da en dos o más etapas del desarrollo infantil, que pueden ser continuas o 
discontinuas. Por ejemplo, un menor de edad que es tratado con negligencia por sus cuidadores, se verá más afectado si experimenta estas situaciones no solo en su infancia temprana, o no solo en su etapa pre-escolar o escolar, sino si las experimenta en por lo menos dos de estas etapas, de forma seguida o interrumpida.

Las similitudes entre las condiciones dadas en el maltrato físico y la negligencia, para generar efectos negativos en el desarrollo de un infante, podrían explicarse porque ambas condiciones generan experiencias de aprendizaje emocional que son conscientes. Es decir, los infantes recuerdan haber sido golpeados y abandonados por sus cuidadores. Esto genera un impacto mediado por la memoria episódica personal, desde la cual el infante podría estar construyendo una historia personal de desconfianza en los otros, si estos maltratos se mantienen en dos o más etapas del desarrollo desde las cuales se adquiere consciencia de los eventos acontecidos. Es interesante resaltar que con el abuso sexual sucede algo distinto. Este tipo de maltrato, que suele tener las peores consecuencias emocionales, suele generar un efecto crónico si ocurre solo una o dos veces, en una sola etapa del desarrollo. Sus efectos son mediados por los procesos inconscientes de aprendizaje emocional, los cuales registran este evento altamente aversivo, porque compromete como ninguno la integridad personal del infante. Los estudios del funcionamiento de estructuras cerebrales del sistema límbico (Gluck, 2009) podrían orientar la explicación del por qué infantes que han sido abusados sexualmente en una infancia temprana, antes de desarrollar sus recuerdos episódicos, podrían estar afectados de manera crónica por estos eventos, así ocurrieran una o dos veces solamente.

Importante también resaltar los efectos paradójicos del maltrato físico, si se tienen en cuenta variables como el momento en que inicia y su continuidad en las etapas del desarrollo. Delima y Vampini (2011) encontraron que el maltrato físico, acompañado de negligencia, suele causar más impacto neurofisiológico si se da desde los 6 años de edad, y no antes. Esto en tanto que el maltrato físico parece afectar de forma más directa a circuitos neurales que conectan a la corteza pre frontal con el hipocampo y el cuerpo calloso, por la sobre activación del circuito de respuesta hormonal hipotalámico-pituitario-adrenal, como principal respuesta ante el estrés. Si la historia de maltrato físico se da en la infancia temprana y en la etapa pre-escolar, pero se interrumpe antes de los 6 años de edad, el infante desarrollaría condiciones de resiliencia que difícilmente se darían si el maltrato continúa en la etapa escolar. Importante tener presente 
que desde los 6 años de edad inicia un proceso de desarrollo de las conexiones entre la corteza pre frontal y resto del encéfalo que harían vulnerables a los infantes desde la etapa escolar, frente a condiciones repetidas de maltrato físico y negligencia.

Con respecto a los efectos cognitivos causados por los diferentes tipos de maltrato, es de resaltar que el abuso sexual suele generar efectos distintos a los generados por condiciones crónicas de maltrato físico y de negligencia. El abuso sexual, genera déficits atencionales, pero no ha mostrado generar efectos negativos en el desempeño intelectual y escolar, como sí lo han demostrado los efectos del maltrato físico y la negligencia que se mantienen en varias etapas del desarrollo. Las explicaciones neuropsicológica se hacen relevantes: Mientras que el maltrato físico y la negligencia pueden llegar a afectar procesos de inhibición de la conducta y de empatía hacia el otro, importantes para tareas que requieren control ejecutivo y planeación del comportamiento, el abuso sexual suele afectar es la respuesta emocional condicionada a recuerdos momentáneos vividos en situaciones similares a la del trauma, afectando solo algunos elementos del proceso atencional. Sin embargo, más investigación sobre los efectos diferenciales de los distintos tipos de maltrato es requerida.

Las estrategias de análisis y medición de los efectos del maltrato infantil requieren entonces un claro análisis de qué tipos de maltrato se han dado durante la historia de una persona, cuál ha sido la continuidad o discontinuidad de estos eventos a través de su desarrollo personal y qué efectos diferenciales se podrían esperar. Sin embargo, una aproximación desde el desarrollo no estaría completa sin tener presente una visión contextual y ecológica de los efectos del maltrato, que busque a su vez definir con claridad cuáles son los tipos de maltrato estudiados. Además, los avances que ha tenido en los últimos años el campo de la neurociencia en mostrar qué cambios neurofisiológicos se dan en la corteza cerebral, asociados a historias de tipos específicos de maltrato, son muy significativos. Frente a la problemática de cómo medir con precisión los eventos de maltrato, sus diferentes tipos, y no ver la validez de tales mediciones afectada por el cambio de los recuerdos y los testimonios, la medición fisiológica representa una importante alternativa para ganar precisión, así como la medición de variables como la continuidad o interrupción de los eventos, así como el tipo de perpetrador del maltrato o abuso. 


\section{Referencias}

Alink, L. R., Cicchetti, D., Kim, J., \& Rogosch, F.A. (2012). Longitudinal Associations Among Child Maltreatment, Social Functioning, and Cortisol Regulation. Journal of Developmental Psychology, 48(1), 224-236. Recuperado de http://www.ncbi.nlm.nih.gov/pmc/articles/PMC3400182/

Allegri, R. F. (2000) Atención y negligencia: bases neurológicas, evaluación y trastornos. Revista de Neurología, 30, 491-495. Recuperado de http://www.uninet.edu/neurocon/congreso-1/conferencias/neuropsicologia-2-3.html

American Psychiatric Publishing (en prensa). Posttraumatic Stress Disorder. Página recuperada el 19 de Junio del 2014 de: http://www.dsm5.org/Documents/PTSD\%20Fact\%20Sheet.pdf.

Barrera. M. (2007). Descripción del Perfil Neuropsicológico de una Muestra de Niños Víctimas de Abuso Sexual en la Ciudad de Medellín. Universidad CES, grupo de investigación en Psicología, Salud y Sociedad. Recuperado de http://www.ascofapsi.org.co/documentos/2010/v_catedra/sesion_6/perfil_neuropsicologico.pdf

Beach, S. R., \& Brody, G.H. (2010). Child Maltreatment Moderates the Association of MAOA With Symptoms of Depression and Antisocial Personality Disorder. Journal of Family Psychology, 24(1), 12-20. Recuperado de http://www.ncbi.nlm.nih.gov/pmc/articles/PMC2839928/

Ben-Arieh, A. (2010). Socioeconomic Correlates of Rates of Child Maltreatment in Small Communities. American Journal of Orthopsychiatry, 80(1), 109-114. Recuperado de http://onlinelibrary.wiley.com/ doi/10.1111/j.1939-0025.2010.01013.x/abstract

Belsky, J. (1980). Child Maltreatment. An Ecological Integration. American Psychologist, 35(4), 320-335. Recuperado de http://psycnet.apa.org/psycinfo/1980-12117-001

Belsky, J. (1993). Etiology of Child Maltreatment: A Developmental Ecological Analysis. PsychologicalBulletin, 114(3), 413-434. Recuperado de http://psycnet.apa.org/index.cfm?fa=buy.optionToBuy\&id=1994-09844-001

Byrne, D. G. et al (2011). Stressor Experience in Primary School-Aged Children: Development Scale to Asses Profiles of Exposure and Effects on Psychological Well-Being. International Journal of Stress Management, 18(1), 88-111. Recuperado de http://psycnet.apa.org/journals/str/18/1/88/ 
Chorpita, B. \& Barlow, D. (1998). The Development of Anxiety: The role of control in the early environment. Psychological Bulletin, 124(1), 3-21. Recuperado de http://www.childfirst.ucla.edu/1998\%20Development\%20of\%20Anxiety.pdf

Degregorio, L. J. (2012) Traumatology. Vol. 19 (2) 158 -166.

Delima, J. \& Vimpani, G. (2011). The neurobiological effects of childhood maltreatment. An often overlooked narrative related to the long-term effects of early childhood trauma?. Family Matters, 89. Recuperado de http://www.aifs.gov.au/institute/pubs/fm2011/fm89/fm89e.html

Diario El Nuevo Siglo (marzo 18 de 2013). Alarmantes cifras del maltrato infantil. Recuperado de http://www. elnuevosiglo.com.co/articulos/3-2013-alarmantes-cifras-de-maltrato-infantil-icbf.html

Erikson, E. (1950; última edición 2000). El Ciclo Vital Completado. Barcelona: Ediciones Paidós Ibérica

Gluck, M. (2009). Aprendizaje y Memoria: Del cerebro al comportamiento. México: McGraw-Hill.

Gracia, E. (1993). El Maltrato Infantil. Un Análisis Ecológico de los Factores de Riesgo. Ministerio de Asuntos Sociales. Madrid, España.

Gracia, E. (2002). El maltrato infantil en el contexto de la conducta parental: Percepciones de padres e hijos. Psicothema, 14(2)274-279. Recuperado de http://www.psicothema.com/pdf/720.pdf

Heyman, R. E., \& Smith, A. M. (2006). Creating and Field-Testing Diagnostic Criteria for Partner and Child Maltreatment. Journal of Family Psychology, 20(3), 397-408. recuperado de http://psycnet.apa.org/index.cfm?fa=buy.optionToBuy\&id=2006-10939-007

Jaffe, S. (2004). The Limits of Child Effects: Evidence for Genetically Mediated Child Effects on Corporal Punishment but Not on Physical Maltreatment. Journal of Developmental Psychology, 40(6), 1047-1058. Recuperado de http://www.ncbi.nlm.nih.gov/pubmed/15535755

Jaffee, S. \& Kohn, A. (2011). Effects of chronic maltreatment and maltreatment timing on children's behavior and cognitive abilities. The Journal of Child Psychology and Psychiatry, 52(2), 184-194. Recuperado de http://www.ncbi.nlm.nih.gov/pubmed/20735512

E 110 
Kaplow, J. B., \& Widom, C. S. (2007). Age of onset of child maltreatment predicts long-term mental health outcomes. Journal of Abnormal Psychology, 116(1), 176-187. Recuperado de http://www.ncbi.nlm.nih. gov/pubmed/17324028

Kinard, E. (2004). Methodological Issues in Assesing The Effects of Maltreatment Characteristics on Behavioral Adjusment in Maltreated Children. Journal of Family Violence, 19(5), 303-318. Recuperado de http:// link.springer.com/article/10.1023\%2FB\%3AJOFV.0000042080.98027.ae

Klenberg. M., Korkman. P., Latí - Nuuttila. (2001). Differential Development of Attention and Executive Functions in 3 - to 12 - Year - Old Finnish Children.

Kosslyn, S. \& Smith, E. (2008). Procesos Cognitivos y Modelos y bases Neurales. Editorial Pearson Prentice Hall.

Locke, T. F., \& Newcomb, M. D. (2003). Childhood Maltreatment, Parental Alcohol/Drug Related Problems, and Global Parental Dysfunction. Journal of Professional Psychology: Research and Practice, 34(1), 73-79. Recuperado de http://psycnet.apa.org/index.cfm?fa=buy.optionToBuy\&id=2003-04125-011

López-Ibor A., Juan J. \& Valdés Miyar, M. (2002). DSM-IV-TR. Manual diagnóstico y estadístico de los trastornos mentales. Texto revisado. Barcelona: Masson.

Marshal, N. A. (2012). A Clinician's Guide to Recognizing and Reporting Parental Psychological Maltreatment of Children. Professional Psychology: Research and Practice, 43(2), 73-79. Recuperado de http://psycnet.apa.org/journals/pro/43/2/73/

Martin, D. (2009). Psicología Experimental: Cómo hacer experimentos en psicología (70 ed). Ediciones Paraninfo.

McLeod, G. F., Fergusson, D. M., \& Horwood, L. J. (2014). Childhood Physical Punishment or Maltreatment and Partnership Outcomes at Age 30. American Journal of Orthopsychiatry, 84(3), 307-315. Recuperado de http://www.ncbi.nlm.nih.gov/pubmed/24827025

Miller, L. (1872). School Behavior Check List: An Inventory of Deviant Behavior for Elementary School Children. Journal of Consulting and Clinical Psychology, 38(1), 134-144. Recuperado de http://psycnet.apa.org/ psycinfo/1972-25206-001

Muller, R. T. (1995). The Interaction of Parent and Child Gender in Physical Child Maltreatment. Canadian Journal of Behavioural Science, 27(4), 450-465. Recuperado de http://www.researchgate.net/publica- 
tion/232541921_The_interaction_of_parent_and_child_gender_in_physical_child_maltreatment

Muller, R.T., Fitzgerald, H.E., Linda, A.S., \& Sucker, R.A. (1994). Social Support and Stress Factors in Child Maltreatment among Alcoholic Families. Canadian Journal of Behavioural Science, 26(3), 438-461. Recuperado de http://psycnet.apa.org/index.cfm?fa=buy.optionToBuy\&id=1995-09823-001

Muñoz, D. (marzo de 2006). El Maltrato Infantil: Un Problema de Salud Pública. Recuperado de http://www.facultadsalud. unicauca.edu.co/fcs/2006/diciembre/maltrato.pdf

Nikulina, V., \& Widom, C.S. (2013). Child Maltreatment and Executive Functioning in Middle Adulthood: A Prospective Examination. Neuropsychology, 27(4), 417-427. Recuperado de http://www.ncbi.nlm.nih.gov/pubmed/23876115

Oberlander, S.E., Thompson, R., Lewis, T., Proctor, L.J., Isbell, P., English, D.J., Du-bowitz, H., Litrownik, A. J., \& Black, M.M. Childhood Maltreatment, Emotional Distress, and Early Adolescent Sexual Intercourse: Multi-Informant Perspectives on Parental Monitoring. Journal of Family Psychology, 25(6), 885-894. Recuperado de http://www. ncbi.nlm.nih.gov/pubmed/21928888

Rosengber, M.S. (1987). New Directions for Research on the Psychological Maltreatment of Children. American Psychologist, 42(2), 166.-171. Recuperado de http://psycnet.apa.org/index.cfm?fa=buy.optionToBuy\&id=1987-19068-001

Rosseli. M., et. al. (2004). Evaluación Neuropsicológica Infantil (ENI): Una batería para la evaluación de los niños entre 5 y 16 años de edad. Estudio normativo colombiano. Revista de Neurología, 38(8), 720-731. Recuperado de http://psy2. fau.edu/ rosselli/NeuroLab/pdfs/revision.pdf

Salinas, P. (2005). Los Procesos Cognitivos Atencionales: Una aproximación conceptual desde lo social. Revista de Antropología Experimental, 5(1), 1-4. Recuperado de http://www.ujaen.es/huesped/rae/articulos2005/salinas2005.pdf

Tajima, E.A., Herrenkol, T.I., Huang, B., \& Whitney, S.D. (2004). Measuring Child Maltreatment: A Comparison of Prospective Parent Reports and Retrospective Adolescent Reports. American Journal of Orthopsychiatry, 74(4), 424-435. Recuperado de http://www.ncbi.nlm.nih.gov/pubmed/15554804

Villodas, M.T., Litrownik, A.J., Thompson, R., Roesh, S.C., English, D.J., Dubowitz, H., Kotch, J.B., \& Runyan D.K. Changes in Youth's Experiences of Child Maltreatment Across Developmental Periods in the LONGSCAN Consortium. Journal of Psychology of Violence, 2(4), 325-338. Recuperado de http://psycnet.apa.org/journals/vio/2/4/325/

\section{$\boldsymbol{E} \| 112$}




\section{Tipos de maltrato y estudio de sus efectos}

Resumen | El presente escrito es una revisión sobre aproximaciones desarrolladas en la investigación científica para poder comprender los efectos del maltrato en la vida infantil y adolescente, especialmente sobre el funcionamiento cognitivo, emocional y conductual. El escrito inicia retomando la relevancia social de este tema en nuestro medio, para luego abordar las dificultades metodológicas y de investigación que han sido propias, a nivel mundial y local, de este tipo de estudios. Seguidamente, el artículo retoma la necesidad de una visión desde el desarrollo y el punto de vista ecológico, para permitir estudiar los efectos psicológicos del maltrato. Finalmente, el escrito se centra en recapitular cuáles son los principales tipos de maltrato que se han estudiado hoy en día, y el porqué es necesario comprenderlos desde una perspectiva del desarrollo, que sea contextualizada, y que retome los avances dados por los estudios neuro-científicos.

Palabras clave | Maltrato, infancia, emociones, cognición, investigación, neurociencia.

\section{Citation}

Muñoz-Olano, J.F. (2014). Tipos de Maltrato y estudio de sus efectos. Enfoques, 1(1). pp. 92-113.

Recibido: 07/05/2014 | Aceptado: 21/06/12014 